\title{
Spontaneous Formation of Indium-Rich Nanostructures on InGaN(0001) Surfaces
}

\author{
Huajie Chen and R. M. Feenstra \\ Department of Physics, Carnegie Mellon University, Pittsburgh, Pennsylvania 15213 \\ J.E. Northrup \\ Xerox Palo Alto Research Center, 3333 Coyote Hill Road, Palo Alto, California 94304 \\ T. Zywietz and J. Neugebauer \\ Fritz-Haber-Institut der Max-Planck-Gesellschaft, Faradayweg 4-6, D-14195 Berlin, Germany
}

(Received 20 April 2000)

\begin{abstract}
InGaN(0001) surfaces prepared by molecular beam epitaxy have been studied using scanning tunneling microscopy and first-principles total energy calculations. Nanometer-size surface structures are observed consisting of either vacancy islands or ordered vacancy rows. The spontaneous formation of these structures is shown to be driven by significant strain in the surface layers and by the relative weakness of the In-N bond compared to Ga-N. Theory indicates that In will preferentially bind at the edges and interior of the structures, thereby giving rise to an inhomogeneous In distribution at the surface.
\end{abstract}

PACS numbers: 68.35.Bs, 61.16.Ch, 68.55.Jk

Alloying is a common approach to realize materials with new and/or tailored properties. For example, semiconductor alloys of the type $\mathrm{A}_{x} \mathrm{~B}_{1-x} \mathrm{C}$ are widely used, where by changing the composition, $x$, the band gap or the lattice parameter can be tuned. A crucial parameter determining the quality and properties of an alloy is the homogeneity: in most cases alloys with large compositional fluctuations have been regarded as inferior for technological applications. However, recent investigations on $\operatorname{In}_{x} \mathrm{Ga}_{1-x} \mathrm{~N}$ (which is used as active layer in $\mathrm{GaN}$ based optoelectronic devices) showed that fluctuations in the In composition might be actually beneficial and significantly improve the luminescence efficiency [1]. For a controlled application of this phenomenon it is crucial to understand the fundamental mechanisms which give rise to the fluctuations and determine their length scale and shape. Theoretically, investigations on alloy composition variations have focused on both bulk phase separation (spinodal decomposition) as well as surface-induced lateral composition modulation (LCM). The latter effect is believed to originate from coupling to strain variations, which themselves arise from an instability of the surface morphology in a strained system [2,3]. LCM occurs since, in essence, large atoms tend to incorporate at surface sites with large in-plane atomic spacing and small atoms incorporate at sites with small spacing. The spatial extent of the strain variations, and presumably of the LCM, varies over a wide range scaling with the inverse square of the average strain [4].

In this work we present a new mechanism for the formation of nanometer-size compositional fluctuations on an alloy surface. Referring specifically to $\operatorname{In}_{x} \mathrm{Ga}_{1-x} \mathrm{~N}$ alloys, the new mechanism occurs because In- $\mathrm{N}$ bonds are so much weaker than $\mathrm{Ga}-\mathrm{N}$ bonds $[5,6]$. The resulting preference for minimizing the number of In-N bonds leads to surface segregation, producing a strained In-rich surface. We observe that an array of surface pits, which we refer to as vacancy islands, forms to relieve the surface strain. Moreover, we find that it is energetically favorable for In atoms to laterally segregate to the edges and interior of the vacancy islands to sites where they form only 0,1 , or 2 bonds with $\mathrm{N}$ atoms compared to 4 bonds for bulk incorporation or 3 bonds for incorporation in sites on the (0001) growth surface. This lateral segregation results in a higher In concentration in and at the edges of the vacancy islands, thus giving rise to In compositional fluctuations at the surface.

The films were grown in a molecular-beam epitaxy (MBE) chamber with base pressure less than $1 \times$ $10^{-10}$ Torr, on Si-polar $6 \mathrm{H}-\mathrm{SiC}(0001)$ substrates. Substrate preparation and MBE film growth are described in Refs. [7] and [8], respectively. Growth was monitored using reflection high-energy electron diffraction, and surfaces were characterized using scanning tunneling microscopy (STM) and Auger spectroscopy. High-resolution $\mathrm{x}$-ray diffraction was used for determining indium incorporation in the films. Total energies were obtained using first-principles theoretical methods, as described in Ref. [6].

Figure 1 shows STM results for the $\operatorname{InGaN}(0001)$ surfaces. The film shown in Fig. 1(a) was grown at $610{ }^{\circ} \mathrm{C}$, with $\mathrm{Ga}$ and $\mathrm{In}$ flux rates of $1.7 \times 10^{14}$ and $4.8 \times$ $10^{13} \mathrm{~cm}^{-2} \mathrm{~s}^{-1}$, respectively, and has a bulk In concentration of $1 \%-2 \%$. The surface was known from Auger measurements to contain $0.9 \pm 0.2 \mathrm{ML}(1 \mathrm{ML}=1.14 \times$ $10^{15}$ atoms $/ \mathrm{cm}^{2}$ ) of indium. Examining Fig. 1(a), we find several regions of apparently different structure. In the lower right-hand corner is a region of uniform, $1 \times 1$ corrugation. Elsewhere on the surface we find areas of brighter (higher) corrugation, with $1 \times$ and $2 \times$ spacing, and in these regions of brighter corrugation we also observe the presence of the small black vacancy islands. Total energy calculations performed for a large number 

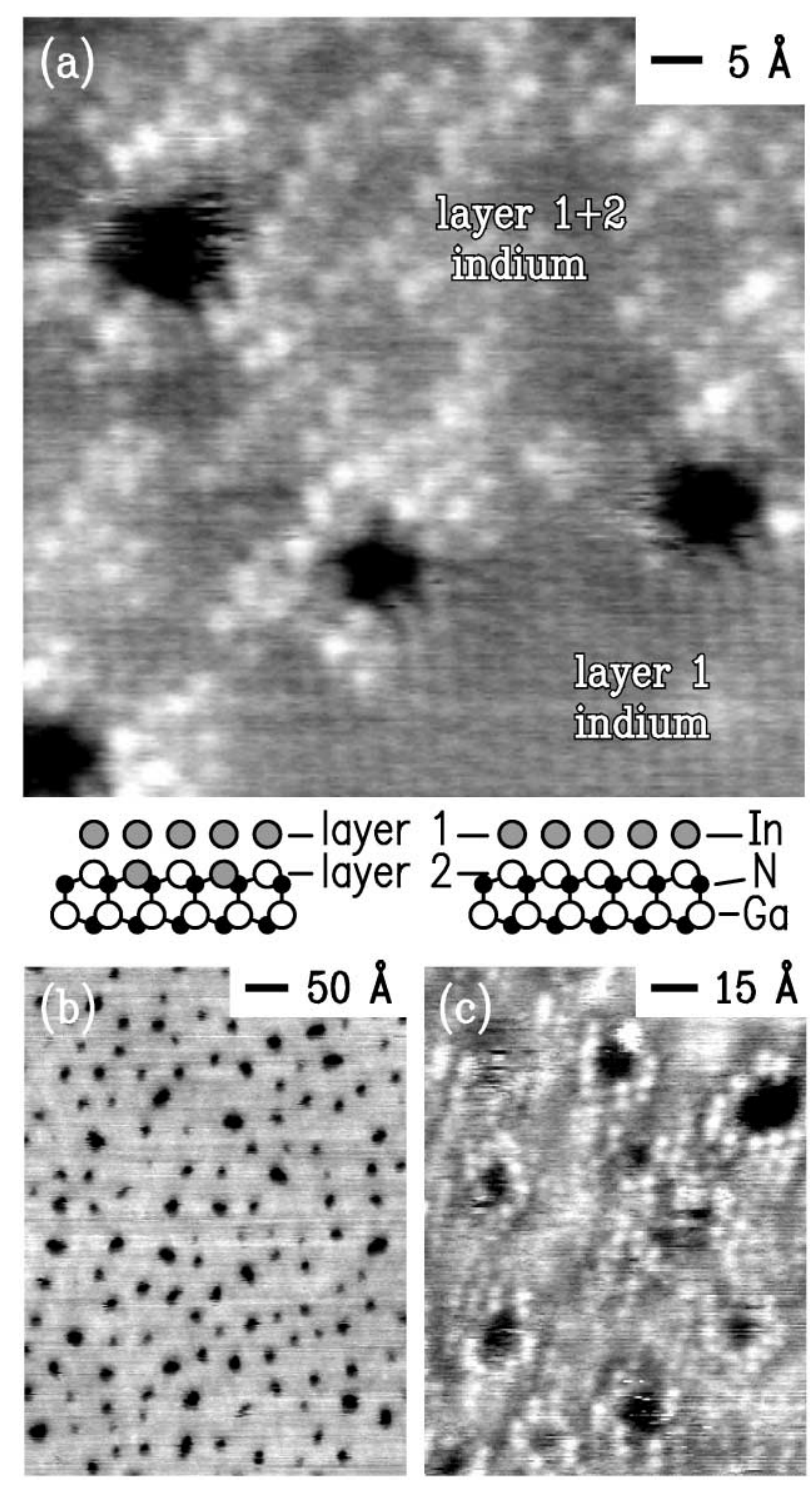

FIG. 1. STM images of InGaN(0001) surface: (a) surface containing $0.9 \pm 0.2 \mathrm{ML}$ of indium. Image was acquired at a sample voltage of $+1.0 \mathrm{~V}$. Grey scale range is $0.6 \AA$. Different regions of the sample are labeled according to their In occupation, layer 1 or layer $1+2$, as pictured below on the image on the right- and left-hand sides, respectively. (b),(c) Surface containing $1.4 \pm 0.2 \mathrm{ML}$ of indium. Images were acquired at sample voltages of (a) $-2.0 \mathrm{~V}$ and $(\mathrm{b})+1.5 \mathrm{~V}$. The entire surface consists of the vacancy island structure. Grey scale range is $0.6 \AA$ for (b) and (c). Tunnel current is $0.075 \mathrm{nA}$ for all images.

of $\operatorname{InGaN}(0001)$ surfaces indicate that a $1 \times 1$ surface with $1 \mathrm{ML}$ of $\mathrm{In}$ in atop sites (layer 1 in Fig. 1) is energetically favorable under In- and Ga-rich conditions [6]. We associate this structure with the $1 \times 1$ region seen in the lower right-hand corner of Fig. 1(a). For more Ga-deficient conditions our calculations show that it becomes energetically favorable to incorporate some In atoms into layer 2 as well. The height of the observed bright maxima, typically $0.2 \AA$ above the nominal height of the $1 \times 1$ region, is consistent with the calculated
$0.3 \AA$ increase in height of a layer 1 atom produced by substitution of In for Ga in layer 2. We therefore attribute the bright corrugation maxima observed elsewhere in the image to the presence of layer 2 In-atoms.

The film pictured in Figs. 1(b) and 1(c) was grown with similar fluxes as that of Fig. 1(a), but at a reduced temperature of $600{ }^{\circ} \mathrm{C}$. It has a bulk In concentration of about $4 \%$ and surface In coverage of $1.4 \pm 0.2 \mathrm{ML}$. In this case the surface phase containing vacancy islands covers the entire surface. The vacancy islands appear dark (lower surface height) for both positive and negative sample bias voltage, indicating that atoms are indeed missing from those areas. The vacancy islands do not grow with time, but rather, they have an equilibrium diameter of 10-20 ̊. The depth of the smallest vacancy islands seen in Fig. 1 is typically $0.8 \AA$, but this value is probably limited by the shape of the STM probe tip. For the larger vacancy islands, we find a depth of about $2.5 \AA$, indicating that at least one layer of atoms is missing from the surface. We find an average separation between vacancy islands of $48 \AA=15$ unit cells, and the vacancy islands cover about $10 \%$ of the total area of the surface. From high resolution images of the regions between vacancy islands, we find a layer 2 In surface coverage there of about $15 \%$. We do not have any experimental measure of the metal composition in the base of the vacancy islands (i.e., In or Ga), but the theory below strongly indicates that those atoms are In. We also note in Fig. 1(c) the presence of bright rings of atoms surrounding the vacancy islands. We associate these bright features with In atoms at the edge of the islands, as revealed in the theoretical analysis below.

At higher In concentrations we observe in addition to the vacancy islands the formation of linear features on the surface, as shown in Fig. 2. This sample was grown with Ga flux of $8.3 \times 10^{13} \mathrm{~cm}^{-2} \mathrm{~s}^{-1}$ and In flux of $1.3 \times$ $10^{14} \mathrm{~cm}^{-2} \mathrm{~s}^{-1}$, and at a growth temperature of $600{ }^{\circ} \mathrm{C}$. We observe vacancy islands similar to those of Fig. 1. In addition, one sees numerous double rows of raised atoms extending along $\langle 11 \overline{2} 0\rangle$ surface directions. These linear features have $2 \times$ periodicity along the rows and nearly $2 \times$ spacing of the bright features across the rows. The corrugation amplitude along the rows is typically $0.2 \AA$, although this value depends on tip-sample voltage.

The array of vacancy islands seen in Figs. 1 and 2 bears some resemblance to that recently observed by Pohl et al. for $\mathrm{Ag}$ monolayers on $\mathrm{Ru}$ [9], and strain energy reduction certainly provides a driving force in both cases [10]. A unique aspect of our case, however, is that lateral segregation of the alloy species on the surface is crucial both for enabling this strain reduction and for reducing the energy by minimizing the number of In-N bonds, as discussed below.

In order to identify the underlying mechanism giving rise to the observed structural instabilities, we have performed first-principles total energy calculations for a variety of $\mathrm{InGaN}(0001)$ surface structures. Because $\mathrm{InGaN}$ 


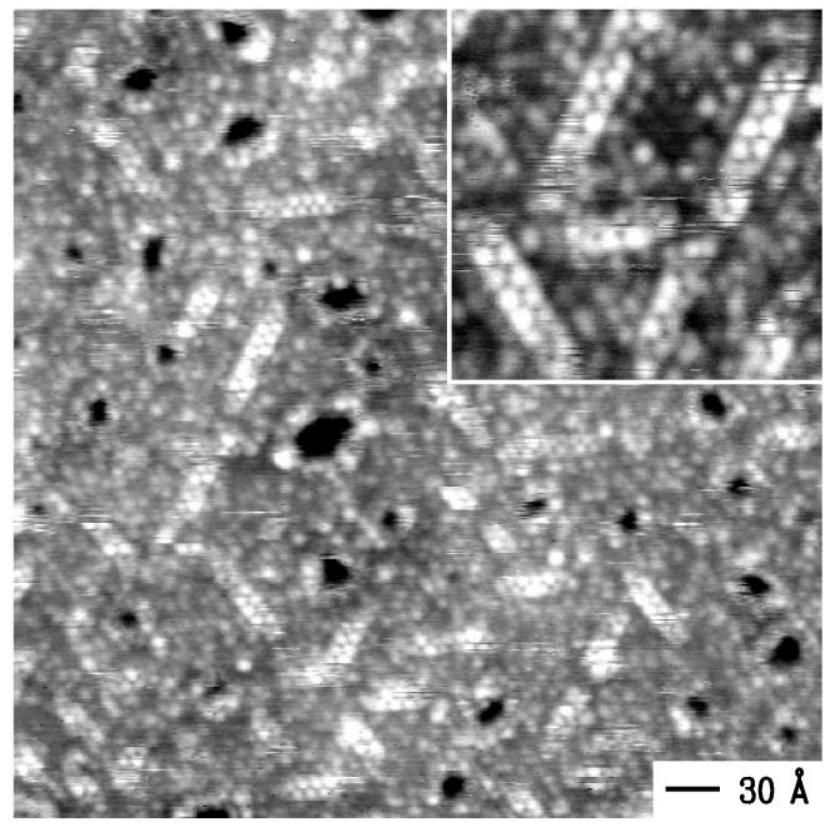

FIG. 2. STM image of high In-content surface, acquired at sample voltage of $+0.8 \mathrm{~V}$ and current of $0.075 \mathrm{nA}$. The inset shows a $2 \times$ expanded view of the double-row features. Grey scale range is $1.4 \AA$ for the main image and $1.0 \AA$ for the inset.

films must be grown under very N-rich (Ga-deficient) conditions to obtain In concentrations greater than a few percent, we focus our attention on surface structures that could be stable under Ga-deficient conditions [6]. Specifically we consider surfaces in which layer 1 is completely occupied by In atoms and layer 2 is occupied by both In and $\mathrm{Ga}$. Structures of this type are indicated schematically in Figs. 1 and 3. Because the In-N bond is $\sim 0.23 \AA$ longer than the Ga-N bond, partial occupation of the second layer by In leads to a substantial surface strain. In the following we show that for sufficiently large In concentrations in layer 2 it is energetically favorable to create $\mathrm{N}$-vacancies in layer 3 . We think this type of structural instability is the fundamental mechanism giving rise to the vacancy islands.

As a first example consider a $2 \times 2$ structure containing $1 \mathrm{ML}$ of In in layer 1 and 3/4 ML of In in layer 2. This structure is denoted $\{7 / 4\}$ and is depicted in Fig. 3(a) [11]. Our total energy calculations show that the creation of a $\mathrm{N}$-vacancy in layer 3 of this structure, as shown in Fig. 3(b), is exothermic. The energy of the reaction $\{7 / 4\} \rightarrow\{7 / 4+\mathrm{N}$-vacancy $\} \quad$ is $\quad \Delta E(\mathrm{eV} /$ vacancy $)=$ $-0.85+\left(\mu_{\mathrm{N}}-\mu_{\mathrm{N} 2}\right)$, where $\mu_{\mathrm{N}}$ is the $\mathrm{N}$ chemical potential and $\mu_{\mathrm{N} 2}$ is the chemical potential of $\mathrm{N}$ in an $\mathrm{N}_{2}$ molecule. Because $\mu_{\mathrm{N} 2}$ is an upper bound on $\mu_{\mathrm{N}}$ the reaction is exothermic by at least $0.85 \mathrm{eV}$ per vacancy. The exothermicity of vacancy formation in a system having a full monolayer of In atoms in layer 2 is even greater: the energy of the reaction $\{8 / 4\} \rightarrow\{8 / 4+\mathrm{N}$-vacancy $\}$ is $\Delta E(\mathrm{eV} /$ vacancy $)=-1.07+\left(\mu_{\mathrm{N}}-\mu_{\mathrm{N} 2}\right)$. One should note that in both of these exothermic reactions each of the three layer 2 sites adjacent to the vacancy is occupied
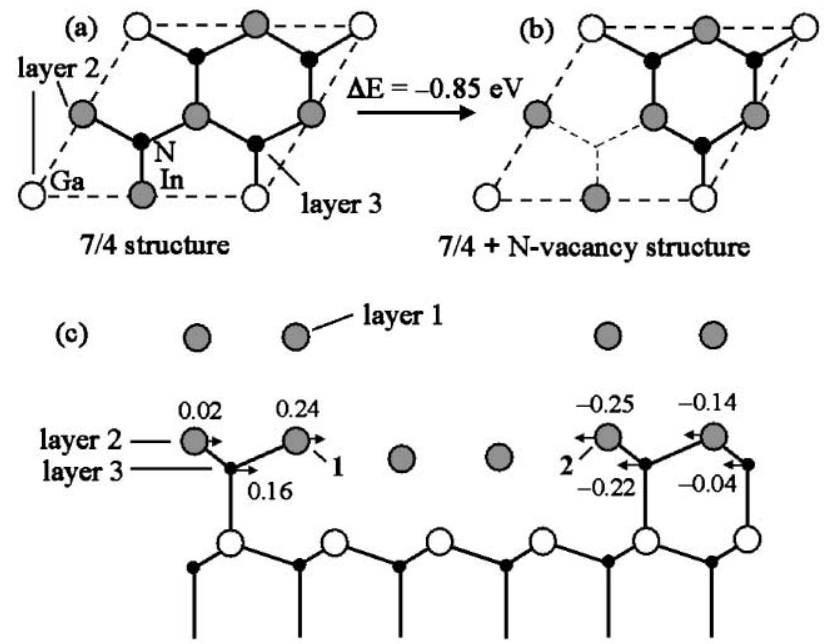

FIG. 3. (a) Top view of the 7/4 structure comprised of 7/4 ML of In. In this $2 \times 2$ structure there is $3 / 4 \mathrm{ML}$ of In and $1 / 4 \mathrm{ML}$ $\mathrm{Ga}$ in layer 2. Layer 1 (not shown) contains $1 \mathrm{ML}$ of In atoms. (b) Top view of the $7 / 4+\mathrm{N}$-vacancy structure, obtained from the $7 / 4$ structure by removing $N$ atoms from layer 3 . (c) Side view of a trench created by removing three rows of $\mathrm{N}$ atoms (layer 3) and two rows of In atoms (layer 1). Formation of this trench leads to substantial lateral displacements of the atoms in layers 2 and 3, shown in $\AA$. Indium atoms in sites 1 and 2 are bonded to 1 and $2 \mathrm{~N}$-atoms, respectively.

by an In atom. In contrast, in the $\{6 / 4+\mathrm{N}$-vacancy $\}$ structure one of the layer 2 sites adjacent to the vacancy is occupied by Ga, and consequently the reaction $\{6 / 4\} \rightarrow$ $\{6 / 4+\mathrm{N}$-vacancy $\}$ is not energetically favorable: $\Delta E(\mathrm{eV} /$ vacancy $)=0.05+\left(\mu_{\mathrm{N}}-\mu_{\mathrm{N} 2}\right)$. These calculations demonstrate that surfaces with a high In concentration are unstable with respect to structural changes that reduce the number of compressively strained In-N bonds. In fact, a homogeneous surface with as little as $1 / 4 \mathrm{ML}$ of In atoms in the layer 2 sites is unstable with respect to vacancy creation. This can be seen by considering the In-conserving reaction in which a $\{5 / 4\}$ structure covering an area $R$ of the surface transforms to a $\{7 / 4+$ $\mathrm{N}$-vacancy\} structure covering an area $R / 3$ together with a $\{4 / 4\}$ structure covering the remaining area $(2 R / 3)$. In this reaction $\mathrm{N}$-vacancies are created and $\mathrm{In}$ atoms segregate laterally to occupy cation sites next to these vacancies. We find such a reaction to be exothermic: the energy change per vacancy is $\Delta E=-0.1 \mathrm{eV}+\left(\mu_{\mathrm{N}}-\mu_{\mathrm{N} 2}\right)$. Thus, as the layer 2 In concentration increases beyond $\sim 1 / 4 \mathrm{ML}$, strain relieving structures are formed in which In atoms occupy the sites having reduced $\mathrm{N}$ coordination.

Given these results it is quite plausible that vacancy islands form in order to relieve surface strain and permit the segregation of In atoms to sites where they exhibit reduced $\mathrm{N}$ coordination, i.e., around the edges of the vacancy islands. Although we cannot confirm this idea directly for the large vacancy island structures seen in the experiment, we can calculate the energies of reactions in which essentially the same type of local bonding transformations and 
strain relief mechanisms are operative. Specifically, we have employed more manageable unit cell dimensions to perform calculations for an array of trenches. As shown in Fig. 3(c), the trenches in this model system are created by removal of rows of layer $3 \mathrm{~N}$-atoms and layer $1 \mathrm{In}$-atoms. Total energy calculations employing a $6 \times 1$ unit cell to model such an array of trenches indicate that trench formation is exothermic and leads to strain relief.

The energy of the reaction in which a $6 \times 1$ unit cell of the $\{8 / 4\}$ structure is transformed to the trench structure shown in Fig. 3(c) is $\Delta E(\mathrm{eV} /$ cell $)=-1.0+$ $3\left(\mu_{\mathrm{N}}-\mu_{\mathrm{N} 2}\right)+2\left(\mu_{\mathrm{In}}-\mu_{\mathrm{In}(\mathrm{bulk})}\right)$. Since $\mu_{\mathrm{N}}<\mu_{\mathrm{N} 2}$ and $\mu_{\text {In }}<\mu_{\text {In(bulk) }}$ this reaction is exothermic. This result implies that the strain relief that is achieved in this transformation outweighs the energy cost of breaking the compressively strained In-N bonds. Indeed, as shown in Fig. 3(c), the existence of the trench allows the layer 2 In- and layer $3 \mathrm{~N}$-atoms to undergo a substantial lateral relaxation. The formation of the trench creates edge sites [sites 1 and 2 in Fig. 3(c)] where In atoms are bonded to just 1 or $2 \mathrm{~N}$-atoms. We propose that the same type of local atomic bonding is present at the edges and interior of the vacancy islands, and this calculation supports the validity of such a model for the vacancy island structure.

Finally, we return to the double-row features seen in Fig. 2, for a surface with high layer 2 In concentration. A possible model for these features consists of a $2 \times$ ordered array of $\mathrm{N}$ vacancies in a region where (locally) the layer 2 In concentration is $\sim 1 \mathrm{ML}$. As seen schematically in Fig. 4 some of the In atoms in layer 2 are adjacent to $\mathrm{N}$-vacancies while some are bonded to three $\mathrm{N}$-atoms. The $2 \times$ corrugation arises from an inequivalence of layer 2 In-atoms since some are bonded to three $\mathrm{N}$ and others to two $\mathrm{N}$-atoms. The former are $0.12 \AA$ higher, giving rise to a height difference of the layer 1 In-atoms of about $0.05 \AA$.

In conclusion, we observe the spontaneous formation of nanometer-size compositional inhomogeneities on In$\mathrm{GaN}(0001)$ surfaces. The structures form to relieve strain of the In-rich surface by the formation of $\mathrm{N}$ vacancies. Substantial energy reduction arises from lateral segregation of In atoms to the edges and interior of the nanostructures. The resultant In concentration in layer 2 shows modulations over a length scale of about $5 \mathrm{~nm}$. Fluctuations on this length scale have indeed been observed in experiments [12] and have been shown to dramatically enhance the luminescence efficiency [1]. Although bulk phase separation in $\mathrm{InGaN}$ has been observed at relatively high temperatures or for thick films [13], the observed effects in thin InGaN quantum wells appear to have different origin. If the bulk In concentration is roughly proportional to the layer 2 In concentration, then the surface reconstruc-

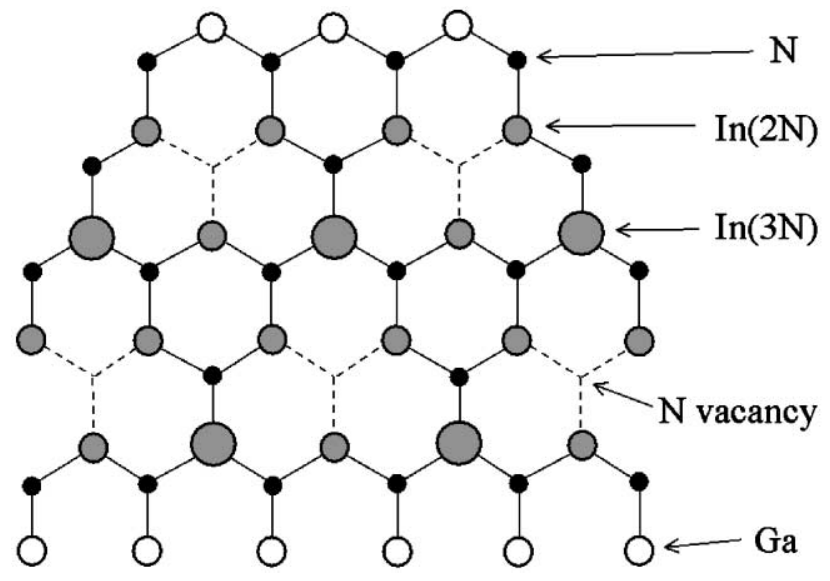

FIG. 4. Top view of a possible model for the $2 \times$ double-row structures. Layer 2 In- and Ga-atoms are shown, together with layer $3 \mathrm{~N}$-atoms. Layer 1 (not shown) contains $1 \mathrm{ML}$ of In atoms. The layer $2 \mathrm{In}$-atoms are bonded to either $2 \mathrm{~N}$-atoms $(2 \mathrm{~N})$ or $3 \mathrm{~N}$-atoms $(3 \mathrm{~N})$.

tion established here provides a new mechanism to produce variations in the bulk In concentration on a nanometer scale.

We are grateful to D.W. Greve for his contributions to this work. The work was supported by the National Science Foundation, Grant No. DMR-9615647, and the Office of Naval Research, Grant No. N00014-96-1-0214 (monitored by Colin Wood).

[1] S. Nakamura, Science 281, 956 (1998).

[2] F. Léonard and R. C. Desai, Phys. Rev. B 57, 4805 (1998), and references therein.

[3] D. J. Srolovitz, Acta Metall. 37, 621 (1989).

[4] A. J. Pidduck et al., Thin Solid Films 222, 78 (1992).

[5] J.E. Northrup, J. Neugebauer, and L. T. Romano, Appl. Phys. Lett. 74, 2319 (1999).

[6] J. E. Northrup and J. Neugebauer, Phys. Rev. B 60, 8473 (1999).

[7] V. Ramachandran et al., J. Vac. Sci. Technol. A 17, 1289 (1999).

[8] H. Chen et al., MRS Internet J. Nitride Semicond. Res. 4S1, G9.5 (1999).

[9] K. Pohl et al., Nature (London) 397, 238 (1999).

[10] K.-O. Ng and D. Vanderbilt, Phys. Rev. B 52, 2177 (1995).

[11] In our notation a $\{n / 4\}$ structure is one in which the total In coverage is $n / 4$, with $1 \mathrm{ML}$ In in layer 1 and $(n-$ 4)/4 ML In in layer 2 . For example, a $\{5 / 4\}$ structure has $1 \mathrm{ML}$ of In in layer 1 and 1/4 ML In in layer 2.

[12] Y. Narukawa et al., Appl. Phys. Lett. 70, 981 (1997).

[13] M. D. McCluskey et al., Appl. Phys. Lett. 72, 1730 (1998). 